\title{
Latent heat of the first-order magnetic transition of $\mathrm{MnFeSi}_{\mathbf{0 . 3 3}} \mathbf{P}_{\mathbf{0 . 6 6}}$
}

\author{
P. Roy, ${ }^{1, *}$ E. Brück, ${ }^{2}$ and R. A. de Groot ${ }^{1}$ \\ ${ }^{1}$ Institute of Molecules and Materials, Faculty of Science, Radboud University, 6525 AJ Nijmegen, The Netherlands \\ ${ }^{2}$ Fundamental Aspects of Materials and Energy, Faculty of Applied Sciences, Delft University of Technology, Mekelweg 15, \\ 2629 JB Delft, The Netherlands
}

(Received 15 June 2015; revised manuscript received 26 January 2016; published 1 April 2016)

\begin{abstract}
The latent heat of a magnetoelastic phase transition is used as a measure of the magnetocaloric effect since it is directly proportional to the entropy change. Taking $\mathrm{MnFeSi}_{0.33} \mathrm{P}_{0.66}$ as a model magnetocaloric material, density functional theory calculations in addition to the phonon calculations based on the density functional perturbation theory were performed in order to calculate the latent heat of the magnetoelastic phase transition. The Curie temperature $\left(T_{C}\right)$ was determined by taking into account the quasiharmonic approximation and the configurational entropy. The material exhibits a first-order magnetic transition accompanied by a large latent-heat $(19.97 \mathrm{~kJ} / \mathrm{kg})$ near-room-temperature operation.
\end{abstract}

DOI: 10.1103/PhysRevB.93.165101

\section{INTRODUCTION}

In recent years, magnetic refrigeration has become one of the most explored fields of research in magnetic materials [1]. The technique is widely accepted to have the potential of replacing the current cooling technology for several reasons. The absence of greenhouse gas refrigerants, the high efficiency, the low noise level, and, in the case of transition-metalbased refrigerants, the expectation of reasonable costs make it very attractive for applications. Magnetic refrigeration relies on the magnetocaloric effect (MCE) discovered by Weiss and Piccard [2] in 1917, which is defined as the reversible change of the magnetic entropy or temperature by the application or removal of an external magnetic field. Until recently, the MCE was only used to achieve sub-Kelvin temperatures $[3,4]$ in a laboratory environment and for space applications. The discovery of giant $\mathrm{MCE}$ in $\mathrm{Gd}_{5} \mathrm{Si}_{2} \mathrm{Ge}_{2}$ by Pecharsky and Gschneidner [5] initiated an extensive search for materials suitable for near-room-temperature applications. Since this discovery, several classes of materials including $\operatorname{MnFe}\left(\mathrm{P}_{1-x} \mathrm{As}_{x}\right)$ [6], $\mathrm{La}(\mathrm{Fe}, \mathrm{Si})_{13}$ [7,8], and their hydrides [9], $\mathrm{Mn}(\mathrm{As}, \mathrm{Sb}), \mathrm{FeRh}$ [10], Heusler alloys [11], and $\mathrm{Mn}_{2} \mathrm{Sb}$ [12] have been proposed as promising candidates for magnetic refrigerants. Some of the materials exhibiting a giant magnetocaloric effect, viz., $\mathrm{La}(\mathrm{Fe}, \mathrm{Si})_{13}$ and $\mathrm{MnFe}\left(\mathrm{P}_{1-x} T_{x}\right)$ [with $T=\mathrm{Si}$, Ge, and As], can be tuned for minimal hysteresis loss around the phase transition, which is necessary for its cyclic operation. The lower hysteresis is, in particular, important when aiming at operation in low magnetic fields below $1 \mathrm{~T}$, making $\mathrm{La}(\mathrm{Fe}, \mathrm{Si})_{13^{-}}$and $\mathrm{MnFe}\left(\mathrm{P}_{1-x} T_{x}\right)$-based materials most promising for real-life applications $[13,14]$. The large MCE in cubic $\mathrm{LaFeSi}_{13}$-based materials is associated with the temperature- and field-induced metamagnetic transition, which comes along with a $1.5 \%$ volume change, whereas the hexagonal $\mathrm{MnFe}\left(\mathrm{P}_{1-x} T_{x}\right)$-based material displays a temperature- and field-induced metamagnetic transition that is accompanied by a significant change in the $c / a$ ratio but hardly any changes in the volume.
For such magnetoelastic transitions, the changes in entropies from both magnetic and elastic transformation at the Curie temperature $\left(T_{C}\right)$ add up and give rise to a larger entropy change. The underlying mechanism was explained recently for $\mathrm{MnFeSi}_{0.5} \mathrm{P}_{0.5}$ using density functional theory calculations $[15,16]$. The coexistence of strongly magnetic atoms and weakly magnetic atoms in the same material (mixed magnetism) gives the characteristic of room temperature $T_{C}$ as well as an enhanced isothermal entropy change in this material. Similar moment instability has recently been reported for $\mathrm{La}(\mathrm{Fe}, \mathrm{Si})_{13}$ [17]. During the past decade, detailed experimental investigations were carried out on $\mathrm{Fe}_{2} \mathrm{P}$-based magnetocaloric materials in order to obtain highly efficient devices [18-21]. The magnitude of MCE in these materials is generally measured by the adiabatic temperature change or the isothermal entropy change at the Curie temperature. Since the system under discussion undergoes a first-order isothermal phase transition, the latent heat $(L)$ is directly proportional to the above two quantities. Hence the evaluation of $L$ is essential for determining the usefulness of magnetocaloric materials for practical application [22]. The latent heat is related to the magnetoelastic transition at $T_{C}$, which depends upon the magnetic entropy change as well as the energy change due to the discontinuous elastic transition, originating from changes in electronic structure and phonon spectrum. An accurate determination of free energies for both phases-below and above the transition-is essential to find $L$ as well as $T_{C}$. A complete set of $a b$ initio calculations taking finite-temperature effects into account can show simultaneously the driving mechanism for the magnetoelastic transition by means of electronic redistribution near the phase transition $[15,16]$ as well as the quantitative value of the parameters (latent heat, entropy change, etc.) obtained. In this paper, we report the phase-transition temperature for $\mathrm{MnFeSi}_{0.33} \mathrm{P}_{0.66}$ determined by accurate phonon calculations, and the latent heat of the metamagnetic translation. These calculated results are in good agreement with the experimental findings.

\section{COMPUTATIONAL DETAILS}

We used the Vienna $A b$ initio Simulation Package (VASP) [23], employing the projector augmented wave (PAW) 
method. Exchange interactions were taken into account using the generalized gradient approximation (GGA) by Perdew, Burke, and Ernzerhof (PBE) [24]. For all of the calculations, PAW data sets were used with $1 s, 2 s, 2 p$, and $3 s$ core states frozen for $\mathrm{Mn}$. In Fe atoms, an additional $3 p$ semicore state was kept frozen, since it lies deeper in energy. For Si and P, the $1 s, 2 s$, and $2 p$ core states were kept frozen. The Brillouin zone integration was performed on a $\Gamma$-centered $k$-point mesh of $6 \times 6 \times 12$ points in the irreducible part of the Brillouin zone. The kinetic-energy cutoff of the plane-wave function was taken as $350 \mathrm{eV}$ and a Gaussian function was used for smearing.

The $\mathrm{MnFeSi}_{x} \mathrm{P}_{1-x}$ series of materials (for $0.3 \leqslant x \leqslant 0.7$ ) crystallizes in the $\mathrm{Fe}_{2} \mathrm{P}$-type layered hexagonal structure with space group $189 / P \overline{6} 2 m$. For this study, we have chosen the $\mathrm{MnFeSi}_{0.33} \mathrm{P}_{0.66}$ composition in order to reserve each crystallographic site for different chemical species within a minimal unit cell, without introducing partial occupancy or large supercells. The Fe atoms prefer to occupy the $3 f$ tetrahedron positions and $\mathrm{Mn}$ atoms prefer the square pyramidal $3 g$ positions [21] and comprise different alternative layers. The partial occupancy $[15,16]$ at the nonmagnetic sites is avoided since it demands larger supercells. We expect minimal effects of positional disorder on the magnetocrystalline transition and utilize the crystallographically ordered model [25] with $\mathrm{Si}$ and $\mathrm{P}$ atoms positioned at $1 b$ and $2 c$ sites, respectively [20]. Our primary aim was to study the phase transition at $T_{C}$, so two different magnetic configurations were set up. The ferromagnetic ordering has a single unit cell containing 9 atoms, where the paramagnetic state was modeled using antiferromagnetic arrangements within the $X Y$ plane with 36 atoms in the supercell. The lattice parameters and atomic positions were both optimized using a criterion for force convergence of $1 \mathrm{meV} / \AA$, and the energies and eigenvalues were converged to $0.01 \mathrm{meV}$. The final lattice parameters obtained are given in Table I.

After obtaining the equilibrium lattice constants, the structures were further relaxed for phonon calculations until $0.01 \mathrm{meV} \AA$ for force and $0.01 \mu \mathrm{eV}$ for energy/eigenvalue convergence. The phonon calculations were done using the finite-difference method as well as density functional perturbation theory (DFPT) [26] as implemented in VASP. In the finite-difference method, one atom was displaced from its equilibrium position and the corresponding forces on all other atoms were calculated. A full set of such forces corresponding

TABLE I. Optimized lattice parameters: $a$ and $c$, atomic positions $3 f \mathrm{Fe}\left(x_{1}, 0,0\right) ; 3 g \mathrm{Mn}\left(x_{2}, 0,1 / 2\right)$, and volume $(V)$ of $\mathrm{MnFeSi}_{0.33} \mathrm{P}_{0.66}$. The experimental parameters [19] were also given for temperature below and above $T_{C}$.

\begin{tabular}{lccccc}
\hline \hline & $a(\AA)$ & $c(\AA)$ & $V\left(\AA^{3}\right)$ & $x_{1}$ & $x_{2}$ \\
\hline Ferromagnetic: & & & & & \\
GGA-PBE & 6.128 & 3.273 & 106.375 & 0.265 & 0.598 \\
Expt. (5 K) & 6.166 & 3.290 & 109.254 & 0.254 & 0.588 \\
Antiferromagnetic: & & & & \\
GGA-PBE & 5.941 & 3.421 & 104.579 & 0.262 & 0.583 \\
Expt. (400 K) & 6.018 & 3.482 & 108.346 & 0.258 & 0.594 \\
\hline \hline
\end{tabular}

to all displacements utilizing symmetry gave a force-constant matrix. In DFPT, a small perturbation potential was added and then the standard DFT formalism was carried out to obtain the force-constant matrix. The force-constant matrices were finally used to obtain the phonon vibrational frequencies. Comparing results from the finite-difference and DFPT methods, we observe very similar force-constant matrices. However, we employed the DFPT method throughout all the calculations as it is computationally cheaper. The inputs for the phonon calculations were obtained using the PHONOPY utility [27]. For computing the Gibbs free energy $(G)$, we used the quasiharmonic approximation (QHA) [28] and obtained phonon vibrational frequencies for different volumes. The phonon calculations usually require bigger unit cells, so a $2 \times 2 \times 2$ supercell was adopted. Finally, as the paramagnetic state is disordered, a configurational-entropy term has to be taken into account [29].

\section{RESULTS AND DISCUSSIONS}

$\mathrm{MnFeSi}_{x} \mathrm{P}_{1-x}$ (for $0.3 \leqslant x \leqslant 0.7$ ) exhibits a first-order magnetoelastic transition between the ferromagnetic and paramagnetic phase at $T_{C}$. The lattice parameter $a$ decreases and $c$ increases [19-22]. The paramagnetic phase is modeled using an antiferromagnetic alignment. To obtain the lowest-energy configuration, different arrangements of magnetic moments were considered. The first one we chose is a $1 \times 1 \times 2$ supercell with an antiferromagnetic order along the $c$ direction. We also consider $2 \times 2 \times 1,2 \times 2 \times 2$, and $2 \times 2 \times 4$ supercells with an antiparallel magnetic alignment between the neighboring unit cells within the $a b$ plane. Accurate energy calculations identify the $2 \times 2 \times 1$ supercell to have the lowest energy. The local moments for the Fe atoms in those configurations decrease from bigger unit cells to smaller unit cells (1.1, 0.9, 0.6 , and $\left.0.5 \mu_{B}\right)$. Experimentally, the local moment for $\mathrm{Fe}$ decreases with the increasing temperature above $T_{C}[18,30]$. This suggests that just above the transition temperature, the paramagnetic state carries a short-range magnetic order, which disappears at higher temperature. Hence, the bigger unit cell corresponds to the state just above $T_{C}$ and the smaller unit cell represents the magnetic state for higher temperatures.

The latent heat of the first-order magnetoelastic phase transition is an important quantity to determine the size of the MCE and hence the effectiveness of the magnetocaloric materials. The amount of heat absorbed during the transition effectively cools down the refrigerator. Theoretically, the latent heat $(L)$ can be calculated from the total entropy change at $T_{C}$. Since the states were modeled at 0 Kelvin using the DFT formalism, a finite-temperature approach is required to obtain the transition temperature $\left(T_{C}\right)$ and hence $L$. The Helmholtz free energy $(F)$ is expressed as the summation of the internal energy $(U)$ in addition to the temperature-dependent term: $F=$ $U-T S$. To evaluate the temperature-dependent term, phonon calculations are necessary. For the lattice vibrational properties of the stable phase, dynamical matrices were created using the DFPT formulation [26]. The eigenvalues and eigenvectors were computed for the dynamical matrices and the phonon vibrational frequencies were obtained using the interatomic force constants. No imaginary phonon frequency was obtained (see Fig. 2), showing vibrational stability of the system for both 

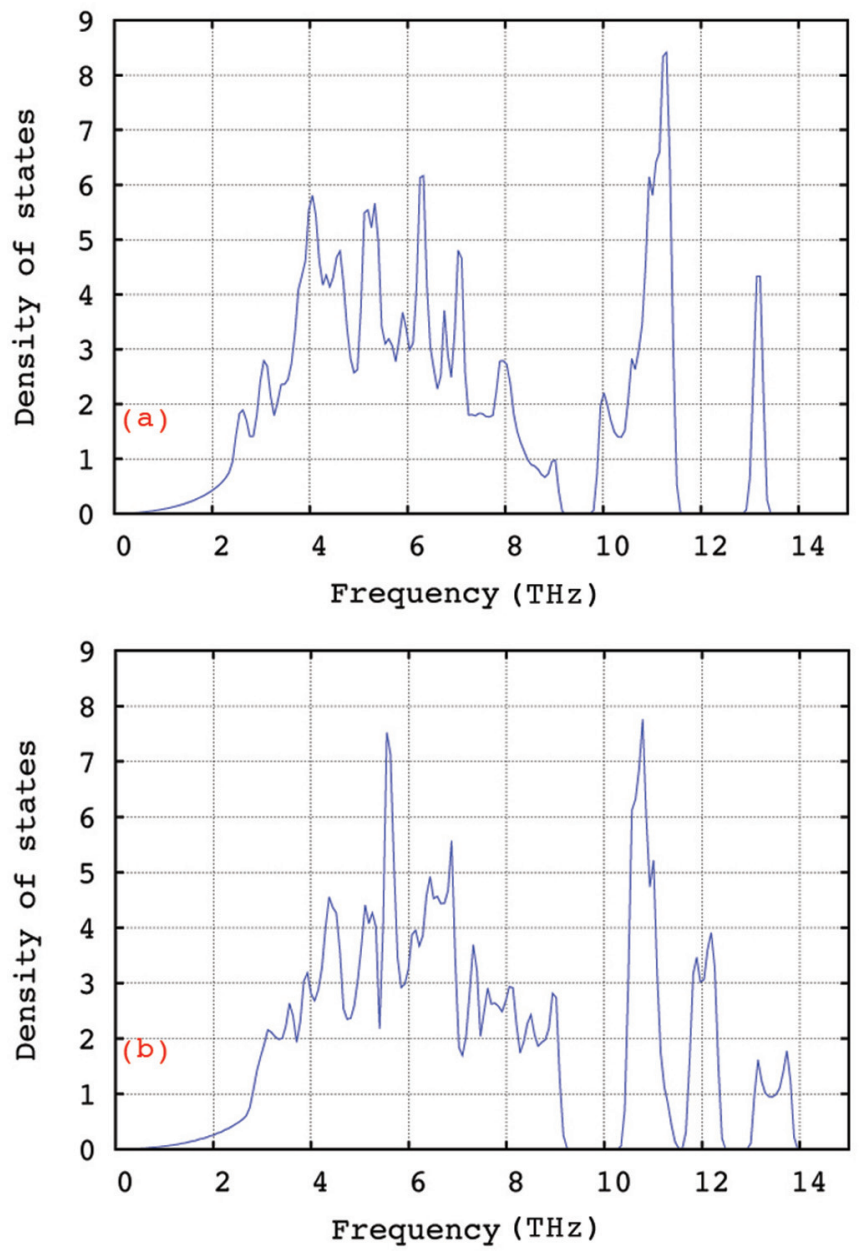

FIG. 1. Phonon density of states of $\mathrm{MnFeSi}_{0.33} \mathrm{P}_{0.66}$ in (a) AFM and (b) FM states as a function of frequency.

magnetic phases. The internal energy term consists of the VASP total-energy output and the zeroth-order phonon vibrational term. So the Helmholtz free energy $F(V, T, m)$ as a function of volume $(V)$, temperature $(T)$, and magnetization $(m)$ takes the following form:

$$
\begin{aligned}
F(V, T, m)= & U_{0}(V, m)+\frac{1}{\Omega_{\mathrm{BZ}}} \sum_{j} \int_{\mathrm{BZ}} \frac{\hbar \omega_{j}(\mathbf{q}, V)}{2} \\
& +k_{B} T \ln \left\{1-\exp \left[\frac{\hbar \omega_{j}(\mathbf{q}, V)}{k_{B} T}\right]\right\} d \mathbf{q} .
\end{aligned}
$$

Here, $\hbar, \omega_{j}(\mathbf{q}, V)$ and $k_{B}$ are the Planck constant, frequency of the $j$ th phonon mode at wave vector $\mathbf{q}$, and the Boltzmann constant, respectively. The phonon density of states and the corresponding band structures for the two magnetic states are shown in Figs. 1 and 2, respectively.

In the density of states as shown in Figs. 1(a) and 1(b), the low-frequency linear parts from 0 to $2.2 \mathrm{THz}$ are similar and represent the acoustic phonons. The optical modes are reasonably different for both of the states above $2.2 \mathrm{THz}$, especially from 8 to $14 \mathrm{THz}$. As shown in Eq. (1), the third term determines the temperature profile of $F(V, T, m)$, which includes the phonon frequencies of the normal modes shown by the phonon DOS. The differences in DOS for
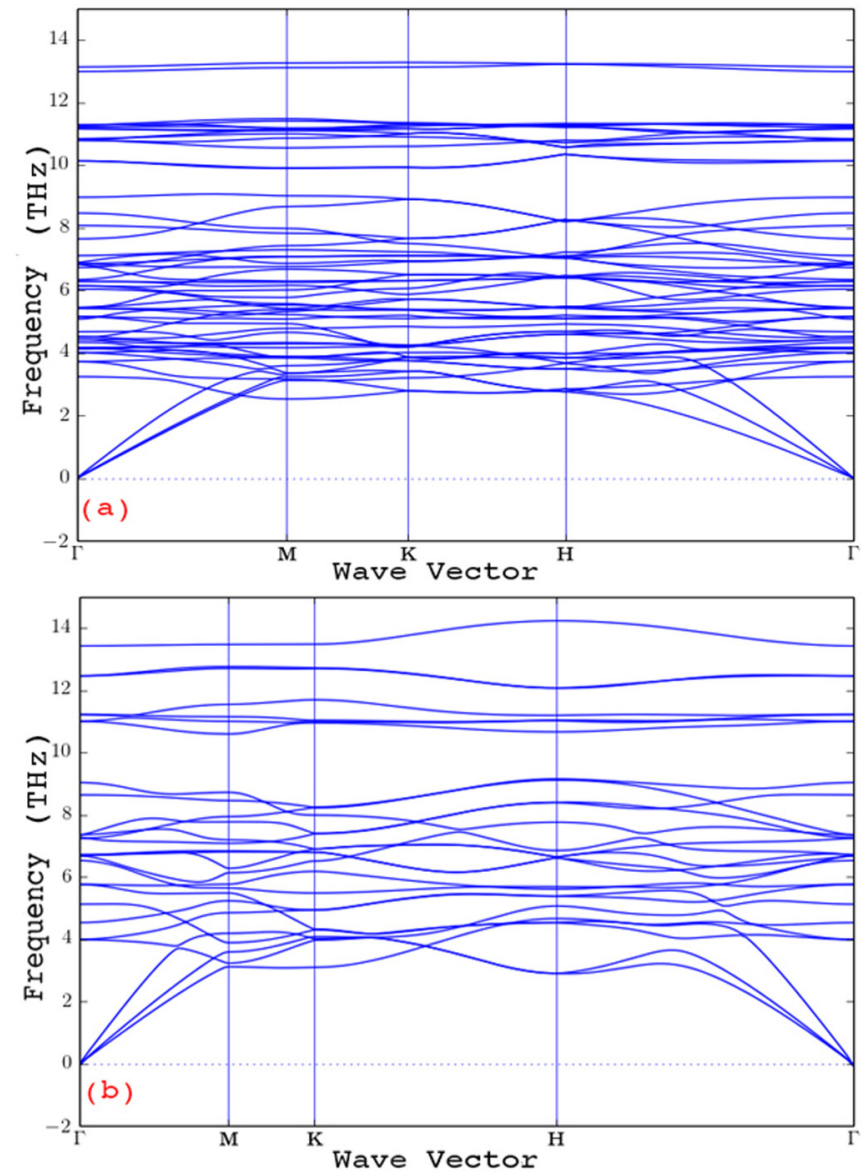

FIG. 2. Phonon band structure of $\mathrm{MnFeSi}_{0.33} \mathrm{P}_{0.66}$ in (a) AFM and (b) FM states.

ferromagnetic (FM) and antiferromagnetic (AFM) states is responsible for the thermal evolution of the free energies that leads to different slopes for the free-energy curves. The low-frequency optical phonon vibrations have a greater contribution to the temperature-dependent third term of Eq. (1) rather than the high-frequency vibrational terms. So, the differences in phonon DOS just above $2.2 \mathrm{THz}$ primarily determine the difference in slopes of the $F$ versus $T$ curves, as shown in Fig. 3.

The FM state represents the ground state at 0 Kelvin, but $F$ decreases gradually for both FM and AFM states with increasing temperature. Despite the fact that the curves have different slopes, they do not intersect even at higher temperature $(\approx 1000 \mathrm{~K})$, which excludes the possibility of any phase transition. This is in direct contradiction with the experimental observation of a magnetoelastic transition at $T_{C}$. However, in the above calculation, we did not consider the volume expansion with temperature. Therefore, to remove this discrepancy in Fig. 3, we introduce the correction for the effect of lattice expansion by incorporating QHA, which includes the volume dependence of the phonon frequencies as a part of the anharmonic effect [28,31]. So, instead of Helmholtz free energy $F(V, T, m)$ for constant volume, we used the Gibbs free energy $(G)$ at constant pressure $(P)$ [32], which is defined as

$$
G(P, T, m)=\min _{V}[F(V, T, m)+P(V, T) V] .
$$




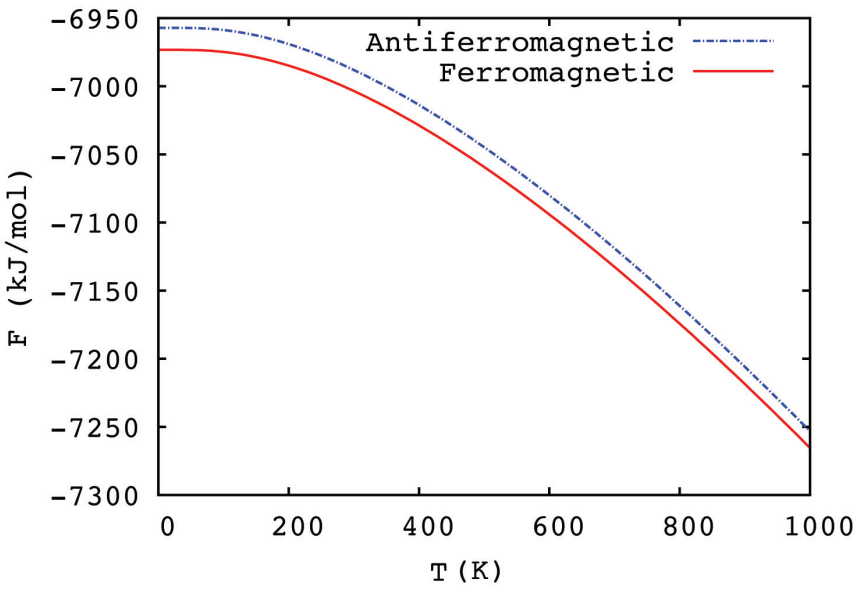

FIG. 3. Helmholtz free energy $(F)$ as a function of temperature.

Following the procedure mentioned by Togo et al. (Fig. 2 of Ref. [33]), $F(V, T, m)$ were evaluated with different volumes for both the FM and the AFM states. Then, fitting $F(V, T, m)$ in the Birch-Murnaghan equation of state, we finally obtain $P(V, T) .{ }^{1}$ From $P(V, T)$ and $F(V, T, m)$, we calculated $G(P, T, m)$ by minimizing the parameters inside the square bracket with respect to the volume [Eq. (2)] [34]. Note that since QHA neglects the temperature dependence of the phonon frequencies, it becomes invalid at very high temperature (well above $1000 \mathrm{~K}$ ).

Additionally, this model does not include the fact that in reality the material in a paramagnetic phase can be assumed to be an assembly of multiple magnetic orders. So in order to include the disorderliness of the system, a configurational entropy term $\left[S_{\text {conf }}=k_{B} \ln W\right]$ was added [29]. Here, $W$ is the number of all possible magnetic configurations. Since a complete magnetically disordered system leads to the Boltzmann limit for the entropy, it is a reasonably good approximation to model a system at high temperature in the paramagnetic phase. Finally, adding all the contributions, the total free energy $\left[F_{\text {tot }}=G(P, T, m)-T S_{\text {conf }}\right]$ was obtained and plotted in Fig. 4.

Experimentally, the Curie temperature lies around $300 \mathrm{~K}$ for $\mathrm{MnFeSi}_{0.33} \mathrm{P}_{0.66}$ [19]. In Fig. 4, the total-free-energy plots intersect at a temperature around $410 \mathrm{~K}$, which depicts the magnetoelastic phase transition and shows good agreement with the experiments. The dissimilarity may arise from other factors contributing to the entropy, such as magnons or phonon-phonon interactions. The difference in $F$ between the FM and AFM state at $410 \mathrm{~K}$ in Fig. 3 is $19.19 \mathrm{~kJ} \mathrm{~mol}^{-1}$. With

${ }^{1}$ The $F(V, T, m)$ versus $V$ was fitted with the Birch-Murnaghan equation of state [38] independently for all of the temperatures,

$$
\begin{aligned}
F(V, T, m)= & -\frac{9}{16} B_{0}\left[\left(4-B_{0}^{\prime}\right) \frac{V_{0}^{3}}{V^{2}}-\left(14-3 B_{0}^{\prime}\right) \frac{V_{0}^{7 / 3}}{V^{4 / 3}}\right. \\
& \left.+\left(16-3 B_{0}^{\prime}\right) \frac{V_{0}^{5 / 3}}{V^{2 / 3}}\right]+ \text { const. }
\end{aligned}
$$

From the fitting, the constants of the equation were obtained. Then the pressure was evaluated using $P(V, T)=-[\delta F(V, T) / \delta V]_{T}$.

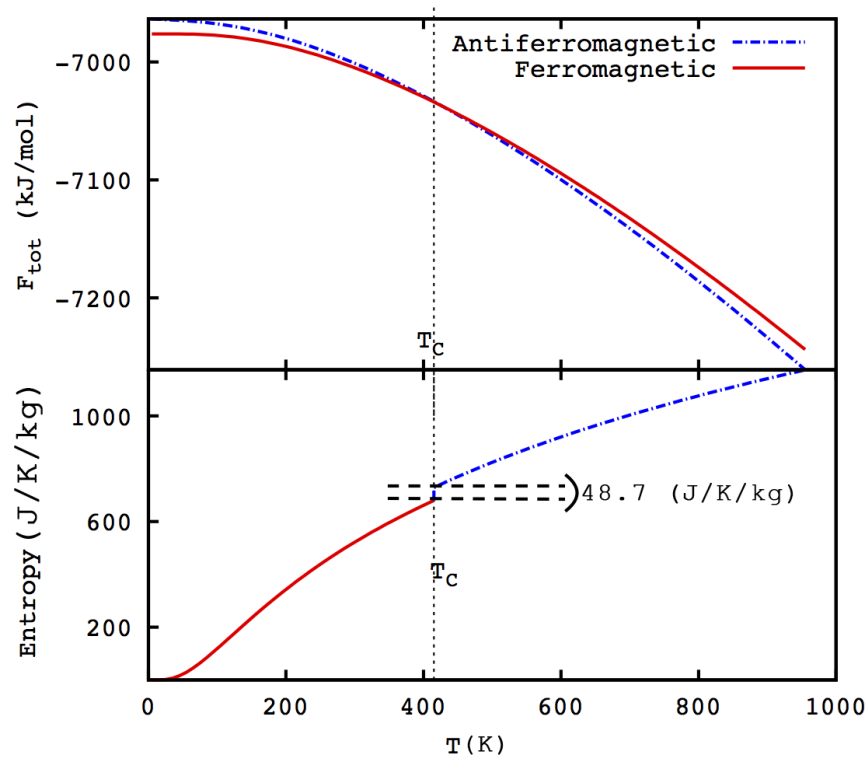

FIG. 4. Total free energy $\left(F_{\text {tot }}\right)$ and entropy $(S)$ evolution with temperature.

the anharmonic correction using Eq. (2), the difference reduces by $6.41 \mathrm{~kJ} \mathrm{~mol}^{-1}$ and decreases further by $12.78 \mathrm{~kJ} \mathrm{~mol}^{-1}$ when including the configurational entropy. The specific heat at constant pressure can also be calculated by deriving the second derivatives of the Gibbs free energy using the relation $C_{P}=-T\left[\delta^{2} G(P, T, m) / \delta T^{2}\right]_{P}$. The specific heat undergoes a discontinuous transition near $T_{C}$ as shown in Fig. 5, which is the characteristic of a first-order phase transition. The entropy as a function of the temperature and the entropy jump at the phase transition $\left(\Delta S_{\text {tr }}\right)$ are shown in Fig. 4. We derive a value of $48.7 \mathrm{~J} \mathrm{~K}^{-1} \mathrm{~kg}^{-1}$, which is comparable to the experimental finding of $58.6 \mathrm{~J} \mathrm{~K}^{-1} \mathrm{~kg}^{-1}$ [35]. The latent heat of the phase transition is defined as $L=T_{C} \times \Delta S_{\mathrm{tr}}$. So the value obtained from this calculation is $19.97 \mathrm{~kJ} \mathrm{~kg}^{-1}$, which is also in good agreement with experimental findings considering the somewhat overestimated value of the Curie temperature $[22,35,36]$. The latent heat is the difference between the slope of $F_{\text {tot }}$ in the FM and AFM state with respect

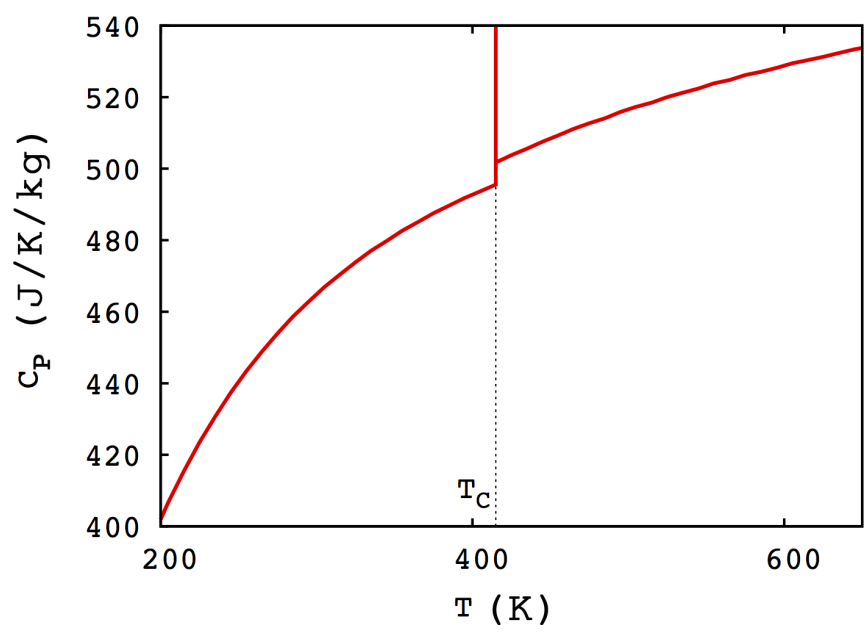

FIG. 5. Calculated $C_{P}$ as a function of temperature. 
to temperature at $T_{C}$. Comparing the latent heat as calculated from the temperature derivative of the total free energy and the Helmholtz free energy at the Curie temperature strongly suggests that the latent heat in this system mainly originates from the configurational entropy change.

\section{CONCLUSION}

Compounds of the type $\mathrm{MnFeSi}_{x} \mathrm{P}_{1-x}$ belong to the MCE materials with the highest magnitude of the MCE (as measured by $\Delta S_{\text {tr }}$ and $\left.\Delta T_{\text {ad }}\right)$ [14]. Our calculation results lead to the following picture. The electronic redistribution that creates covalent bonding among the atoms $[15,16]$ is responsible for both the electronic part of the entropy change and the change in thermal vibrations of the atoms that contributes to the lattice entropy change. In addition to the regular magnetic part of the magnetic-field-induced entropy change at $T_{C}$, this magnetoelastic transition contributes dominantly to the configurational and much less to the lattice part of entropy change. The electronic redistribution initiates the lattice change and both of these contributions add up to a bigger entropy change and a large latent heat with unexpectedly low thermal hysteresis [37]. The latent heat of this system is primarily considered as being of magnetic (configurational) rather than lattice origin, which should also be true for $\mathrm{La}(\mathrm{Fe}, \mathrm{Si})_{13}$ materials, in contrast to the findings of Grüner et al. [17].

In this study, we use the stereotypical compound $\mathrm{MnFeSi}_{0.33} \mathrm{P}_{0.66}$ with $\mathrm{Mn}: \mathrm{Fe}=1: 1$ stoichiometry. To take thermal effects into account, we utilize a $2 \times 2 \times 2$ supercell. The need for such larger supercell sizes restricts the method for low symmetric structures because of high computational costs at the moment. In addition to this, nonmagnetic Si and $\mathrm{P}$ atoms were inserted at crystallographically distinct $1 b$ and $2 c$ sites, respectively, in order to avoid the computational cost related again to larger supercells to account for positional disorder. DFT optimized rather than experimentally determined structures were used for both high and low magnetic states in $\mathrm{MnFeSi}_{0.33} \mathrm{P}_{0.66}$. The temperature evolution of the free energy $(F)$ in these states was evaluated using the efficient DFPT mechanism. Further corrections to the free energy were included in the form of QHA and configurational entropy. We calculate the phase-transition temperature, $\Delta S_{\mathrm{tr}}$, and the latent heat of the system within reasonable accuracy [22,35,36]. The observed dominance of the magnetic (configurational) part of the latent heat is awaiting experimental verification. This method can be used to calculate latent heat or related quantities of other magnetic systems and characterize them based on the size and nature of the MCE in the search for better magnetocaloric materials in the near future.

\section{ACKNOWLEDGMENTS}

This work is financially supported by the Industrial Partnership Program (IPP-I28) of Foundation for Fundamental Research on Matter (FOM) (The Netherlands) and co-financed by BASF New Business. The authors would like to thank Dr. Gilles A. de Wijs, and Dr. Ebrahim Hazrati for helpful discussions.
[1] A. Smith, C. R. H. Bahl, R. Bjork, K. Engelbrecht, K. Nielson, and N. Pryds, Adv. Energy Mater. 2, 1288 (2012).

[2] P. Weiss and A. Piccard, J. Phys. Theor. Appl. 7, 103 (1917).

[3] W. F. Giaque, J. Am. Chem. Soc. 49, 1864 (1927).

[4] P. Debye, P. Ann. Phys. 386, 1154 (1926).

[5] V. K. Pecharsky and K. A. Gschneidner, Jr., Phys. Rev. Lett. 78, 4494 (1997).

[6] O. Tegus, E. Brück, K. H. J. buschow, and F. R. de Boer, Nature (London) 415, 150 (2002).

[7] O. Gutfleisch, A. Yan, and K. H. Muller, J. Appl. Phys. 97, 10M305 (2005).

[8] M. K. Han and G. J. Miller, Inorg. Chem. 47, 515 (2008).

[9] A. Fujita, S. Fujieda, Y. Hasegawa, and K. Fukamichi, Phys. Rev. B 67, 104416 (2003).

[10] S. Nikitin, G. Myalikgulyev, A. Tishin, M. Annaorazov, K. Asatryan, and A. Tyurin, Phys. Lett. A 148, 363 (1990).

[11] T. Krenke, E. Duman, M. Acet, E. F. Wasserman, X. Moya, L. Mañosa, and A. Planes, Nat. Mater. 4, 450 (2005).

[12] L. Caron, X. Miao, J. P. Klaasse, S. Gama, and E. Brück, Appl. Phys. Lett. 103, 112404 (2013).

[13] E. Brück, J. Phys. D: Appl. Phys. 38, R381 (2005).

[14] K. G. Sandeman, Scr. Mater. 67, 566 (2012).

[15] N. H. Dung, Z. Q. Ou, L. Caron, L. Zhang, D. T. Cam Thanh, G. A. de Wijs, R. A. de Groot, K. H. J. Buschow, and E. Brück, Adv. Energy Mater. 1, 1215 (2011).
[16] E. K. Delczeg-Czirjak, M. Pereiro, L. Bergqvist, Y. O. Kvashnin, I. Di Marco, G. Li, L. Vitos, and O. Eriksson, Phys. Rev. B 90, 214436 (2014).

[17] M. E. Gruner, W. Keune, B. Roldan Cuenya, C. Weis, J. Landers, S. I. Makarov, D. Klar, M. Y. Hu, E. E. Alp, J. Zhao, M. Krautz, O. Gutfleisch, and H. Wende, Phys. Rev. Lett. 114, 057202 (2015).

[18] N. H. Dung, L. Zhang, Z. Q. Ou, L. Zhao, L. van Eijck, A. M. Mulders, M. Avdeev, E. Suard, N. H. van Dijk, and E. Brück, Phys. Rev. B 86, 045134 (2012).

[19] Z. Ou, Ph.D. thesis, Delft University of Technology, Delft, 2013.

[20] X. F. Miao, L. Caron, P. Roy, N. H. Dung, L. Zhang, W. A. Kockelmann, R. A. de Groot, N. H. van Dijk, and E. Brück, Phys. Rev. B 89, 174429 (2014).

[21] D. T. Cam Thanh, E. Brück, O. Tegus, J. Klaasse, and K. Buschow, J. Magn. Magn. Mater. 310, e1012 (2007).

[22] F. Guillou, G. Porcari, H. Yibole, N. van Dijk, and E. Brück, Adv. Mater. 26, 2671 (2014).

[23] G. Kresse and J. Furthmuller, Phys. Rev. B 54, 11169 (1996).

[24] J. P. Perdew, K. Burke, and M. Ernzerhof, Phys. Rev. Lett. 77, 3865 (1996).

[25] V. Höglin, M. Hudl, L. Caron, P. Beran, M. H. Sørby, P. Nordblad, Y. Andersson, and M. Sahlberg, J. Solid State Chem. 221, 240 (2015).

[26] S. Baroni, S. de Gironcoli, A. Dal Corso, and P. Giannozzi, Rev. Mod. Phys. 73, 515 (2001). 
[27] A. Togo, F. Oba, and I. Tanaka, Phys. Rev. B 78, 134106 (2008).

[28] N. W. Ashcroft and N. D. Mermin, Solid State Physics (Saunders, Philadelphia, 1976).

[29] C. M. Fang, G. A. de Wijs, E. Orhan, G. de With, R. A. de Groot, H. T. Hintzen, and R. Marchand, J. Phys. Chem. Solids 64, 281 (2003).

[30] H. Yibole, F. Guillou, L. Caron, E. Jiménez, F. M. F. de Groot, P. Roy, R. de Groot, N. H. van Dijk, and E. Brück, Phys. Rev. B 91, 014429 (2015).

[31] N. D. Drummond and G. J. Ackland, Phys. Rev. B 65, 184104 (2002).

[32] M. T. Dove, Introduction to Lattice Dynamics (Cambridge University Press, Cambridge, 2005).
[33] A. Togo, L. Chaput, I. Tanaka, and G. Hug, Phys. Rev. B 81, 174301 (2010).

[34] T. Tsuchiya and J. Tsuchiya, Proc. Natl. Acad. Sci. 108, 1252 (2011).

[35] F. Guillou, H. Yibole, N. H. van Dijk, and E. Brück, J. Alloys Compd. 632, 717 (2015).

[36] H. Yibole, F. Guillou, L. Zhang, N. H. van Dijk, and E. Brück, J. Phys. D: Appl. Phys. 47, 075002 (2014).

[37] K. Gschneidner, Y. Mudryk, and V. K. Pecharsky, Scr. Mater. 67, 572 (2012).

[38] T. Tsuchiya and K. Kawamura, J. Chem. Phys. 117, 5859 (2002). 